\title{
Stromal Vascular Fraction-Enriched Fat Grafting as Treatment of Adherent Scars: Study Design of a Prospective Cohort Study.
}

Linda Vriend ( $\square$ lindavriend@gmail.com )

Universitair Medisch Centrum Groningen https://orcid.org/0000-0002-9439-5810

Joris Anton van Dongen

University of Utrecht: Universiteit Utrecht

Anouk Pijpe

Red Cross Hospital: Rode Kruis Ziekenhuis

Marianne K. Nieuwenhuis

Martini Hospital: Martini Ziekenhuis

Sandra JM Jongen

Martini Hospital: Martini Ziekenhuis

Martin Conrad Harmsen

University of Groningen: Rijksuniversiteit Groningen

Paul PM van Zuijlen

Amsterdam UMC VUMC Site: Amsterdam UMC Locatie VUmc

Berend van der Lei

University of Groningen: Rijksuniversiteit Groningen

\section{Research Article}

Keywords: adherent scars, scar quality, burn scars, Stromal Vascular Fraction, Adipose-derived stromal cells, ASC, fat grafting, pliability

Posted Date: January 19th, 2022

DOI: https://doi.org/10.21203/rs.3.rs-1112004/v1

License: (c) (i) This work is licensed under a Creative Commons Attribution 4.0 International License.

Read Full License 


\section{Abstract}

\section{Background}

In the last decades autologous fat grafting has been used to treat adherent dermal scars. The observed regenerative and scar reducing properties have been mainly ascribed to the tissue-derived stromal vascular fraction (tSVF) in adipose tissue. Adipose tissue's components augment local angiogenesis and mitosis in resident tissue cells. Moreover, it promotes collagen remodeling. We hypothesize that tSVF potentiates fat grafting-based treatment of adherent scars. Therefore, this study aims to investigate the effect of tSVF-enriched fat grafting on scar pliability over a 12-month period.

\section{Methods \& Design}

A clinical multicenter prospective cohort trial will be conducted in two dedicated Dutch Burn Centers (Red Cross Hospital, Beverwijk and Martini Hospital, Groningen). After their consent, 46 patients ( $\geq 18$ years) with adherent scars caused by burns, necrotic fasciitis or degloving injury who have an indication for fat grafting will receive a sub-cicatricic tSVF-enriched fat graft. The primary outcome is the change in scar pliability measured by the Cutometer between pre- and 12 months post-grafting. Secondary outcomes are scar erythema and melanin measured by the DSM II Colormeter, scar quality assessed by the patient and observer scales of the Patient and Observer Scar Assessment Scale (POSAS) 2.0 and histological analysis of scar biopsies and tSVF quality and composition. This study has been approved by the Dutch Central Committee for Clinical Research (CCMO), NL72094.000.20.

\section{Conclusion}

This study will test the clinical efficacy of tSVF-enriched fat grafting to treat dermal scars while the underlying working mechanism will be probed into too.

\section{$\underline{\text { Trial status }}$}

Dutch Trial Register: NL 8461, registered on 16 March 2020.

\section{Background}

The first fat grafting procedure was described in 1893 to treat a soft tissue defect for reconstructive purposes ${ }^{1}$. Nowadays, fat grafting is routinely used for both reconstructive as well as aesthetic purposes and is far 'more than volume correction alone'2,3. In 2001, Zuk et al. described the adipocyte stromal cells (ASCs) that are present in large amounts in the Stromal Vascular Fraction (SVF) of adipose tissue and possess multipotent capabilities ${ }^{5}$. This discovery led to an increased use of fat grafting for regenerative and scar-reducing purposes and to treat damaged tissues, such as burn wounds ${ }^{4}$. Since then, it is thought that observed beneficial effects are for a large part attributed to the SVF in adipose tissue. 
SVF is easily obtained by dissociating lipoaspirate enzymatically or mechanically ${ }^{6}$. SVF comprises the non-adipocyte fraction of fat and is composed of stromal cells such as fibroblasts and other mesenchymal stromal cells as well as vasculature cells (endothelial and smooth muscle). The stromal cells produce and maintain the extracellular matrix that embeds and supports the cells in SVF. Recently, a reliable, fast, intra-operative mechanical isolation procedure has been developed to yield tissue-derived stromal vascular fraction (tSVF), which makes clinical application of tSVF more feasible than enzymatic isolated SVF (cellular SVF) ${ }^{7-9}$. On the one hand tSVF probably effectuates better retention of paracrine factors through extracellular matrix components, that lack in single cell suspensions like cSVF, where cells disappear through leakage of lymph vessels. On the other hand, tSVF is a unit of transplanted tissue that provides a nutritious environment, serving as a therapeutic component of adipose tissue. Case reports and retrospective studies have reported improved aesthetic outcomes and pain relieve after autologous fat grafting of problematic and adherent scars $3,4,10-23$. These results hold promise but, besides the obvious methodological drawbacks of study design, lack adequate scar evaluation with validated objective or subjective scar measurement tools. Moreover, physical, physiological and psychological evaluation and measurements are also important since adherent scars cause physical burden e.g. stiffness and impaired range of motion, and psychological burden e.g. psychosocial distress ${ }^{24,25}$. Several validated scar scales, e.g., the Patient and Observer Scar Assessment Scale (POSAS), Vancouver Scar Scale (VSS) and Manchester Scar Scale (MSS) have been developed to assess the aforementioned aspects of scar quality $26,27,28$. The POSAS is recognized as a highly reliable scar rating scale, moreover it also includes the patients' opinion and is therefore favorable for subjective scar assessment ${ }^{29,30}$.

Recently, autologous fat grafting was effectively used to improve scar quality, in particular scar pliability and pain in adherent scars ${ }^{31}$. Scar pliability and color were assessed with validated objective measures, i.e. the Cutometer and DSM II Colormeter, and scar quality and pain with validated subjective measures, i.e. the POSAS patient and observer scales $29,30,32-37$. The results of the beforenamed studies warrant further research with SVF or ASCs, because these components are deemed responsible for a large part of the observed ameliorating effects on scar features ${ }^{3,4,10-23,31}$. Moreover, enrichment of fat grafts with SVF may induce even larger and longer lasting ameliorating effects on scar features than plain autologous fat.

A well-designed clinical trial investigating these potential effects of SVF-enriched fat grafting is warranted. The aim of this study is to assess the efficacy of tSVF-enriched fat grafting in patients with adherent scars using a comprehensive scar evaluation protocol. This study will also provide benchmark data on histological change of adherent scars after tSVF-enriched fat grafting.

\section{Methods, Design And Outcome Measures}

\section{Objectives}


The aim of this study is to assess the efficacy of tSVF-enriched fat grafting in patients with adherent scars. The primary outcome measure is the change in scar pliability as assessed with the Cutometer Skin Elasticity Meter Dual MPA 580 (Courage and Khazaka GmbH, Cologne, Germany) pre- and 12 months post tSVF-enriched grafting. The secondary outcome measures are scar erythema and melanin index, as a proxy for color and pigmentation, measured by the DSM II Colormeter (Cortex Technology, Hadsund, Denmark), scar quality assessed by the patient and observer scales of the Patient and Observer Scar Assessment Scale (POSAS 2.0), and histological features of tSVF and scar biopsies.

\section{Protocol and registration}

This study was approved by the Dutch Central Committee for Clinical Research (CCMO) NL72094.000.20, and by the Institutional Review Boards of the participating hospitals (Red Cross Hospital, Beverwijk, and Martini Hospital, Groningen, the Netherlands). Methods of the study are specified in a protocol that is registered at the Dutch trial register (March 16th, 2020, NL 8461) ${ }^{38}$.

\section{Trial design}

A prospective intra-patient before-after cohort-study on the effect of tSVF-enriched fat grafting on scar pliability in patients with adherent scars and who are treated in the Red Cross Hospital, Beverwijk, or the Martini Hospital, Groningen, the Netherlands (Figure 1).

\section{Participants}

Patients, aged $\geq 18$ years with adherent scars ( $\geq 12$ months old) caused by burns, necrotic fasciitis or degloving injury, visiting the outpatient clinic of one of the Burn Centers in Beverwijk or Groningen who have an indication for fat grafting are eligible for this trial. Exclusion criteria are: previous scar treatment with fat grafting, skin melanoma in patients' history, pregnant or lactating, BMI <18 (general exclusion criteria for fat grafting procedure), unlikely to comply with the requirements of the study protocol and follow-up, and insufficient knowledge of the Dutch language (Table 1). Patients are included after they received a confirmed understandable and neutral explanation of the study by a member of the research team and after signing informed consent following the guidelines of the CCMO. 
Table 1

Inclusion criteria

- Age $\geq 18$ years

- Patient has an adherent scar (minimum scar age: 12 months) caused by burns, necrotic fasciitis or degloving injury, for which fat grafting is indicated

- Competent adults

- Patients seen by a plastic surgeon in Burn centers of the Red Cross Hospital, Beverwijk or Martini Hospital, Groningen, The Netherlands

\section{Exclusion criteria}

- Previous scar treatment with fat grafting in selected scar

- General exclusion criteria for fat grafting procedure: pregnancy, lactating, $\mathrm{BMI}<18 \mathrm{~kg} / \mathrm{m}^{2}$

- Skin melanoma in patient's history

- Unwillingness to commit to the study protocol and show up for all follow up moments

- Insufficient proficiency in Dutch to the extent that clear communication is not possible

\section{Scar selection}

Before surgery, one scar area will be selected and marked according to a standardized algorithm for objective data collection (Figure 2$)^{31}$. The maximum size of the scar area is limited by the maximum quantity of tSVF that can be obtained and a minimum length of $2 \mathrm{~cm}$ will apply. Each scar consists of five measurement points. The five points in the selected area will be subjected to all study outcomes.

\section{Surgical procedure}

Under general anesthesia, lipoaspirate is harvested from the abdominal wall, flank or thigh with fine harvesting cannulas. Out of $10 \mathrm{ml}$ lipoaspirate, $1 \mathrm{ml}$ of tSVF will be produced intraoperatively with the Fractionation of Adipose Tissue (FAT) procedure, a fat dissociation procedure using a fractionator (Figure 3 and 4$)^{39}$. For preparing the tSVF-enriched fat graft, $10 \mathrm{ml}$ lipoaspirate in a $10 \mathrm{ml}$ Luer-Lock syringe will be centrifuged at 3,000 rpm with a $9.5 \mathrm{~cm}$ radius fixed angle rotor for 2.5 minutes (Medilite, Thermo Fisher Scientific, Waltham, MA) at room temperature (Figure 5). Thereafter, the upper oily layer will be drained from the top while the bottom layer will be drained by removing the lower cap of the syringe. Subsequently in the syringe $9.0 \mathrm{ml}$ of fat graft will be left to which is added $1.0 \mathrm{ml}$ of tSVF: two syringes, one with $9 \mathrm{ml}$ of fat and one with $1 \mathrm{ml}$ of tSVF will be connected to a Luer-Lock and the contents will be mixed by gently pushing de content of one syringe into the other, this way yielding the tSVF-enriched fat. Small incisions at the border of the scar are created to perform adhesiolysis of the selected scar. Then, the tSVF-enriched fat (tenfold excess fat over tSVF) will be injected under the scar. This procedure is repeated until the entire scar area has reached fat grafting saturation (when enriched fat graft starts coming out of the incision holes). After fat saturation, the scar is covered and fixed with gausses.

Practically, $50 \mathrm{ml}$ of fat has to be harvested to yield $25 \mathrm{ml}$ of fat and $2.5 \mathrm{ml} \mathrm{tSVF}$, resulting in $27.5 \mathrm{ml}$ tSVF enriched fat graft.

\section{Study outcomes}




\section{Primary outcome measure}

\section{Scar pliability}

The primary outcome measure is the change in scar pliability as measured by the Cutometer Skin Elasticity Meter Dual MPA 580 ® (Courage and Khazaka GmbH, Cologne, Germany) pre- and 12 months post tSVF-enriched fat grafting (Figure 2 and 6 ). The Cutometer ${ }^{\circledR}$ is a validated, reliable instrument which measures viscoelasticity of the skin by analyzing its maximal extension after inducing negative pressure $^{35,36}$.

\section{Secondary outcome measures}

All scar outcome measurements will be performed by two trained observers who work independently of each other to prevent confirmation bias.

\section{Scar mechanics}

Scar, retraction, elasticity, viscoelasticity and maximum extension will be measured with the Cutometer Skin Elasticity Meter Dual MPA 580 ® (Courage and Khazaka GmbH, Cologne, Germany) pre- tSVFenriched fat grafting and 3 - and 12 months post tSVF-enriched grafting (Figure 2 and 6$)^{31}$. Scar pliability will also be measured after 3 months post tSVF-enriched fat grafting.

Healthy skin measurements will be conducted as described pre-, 3 , and 12 months post-tSVF grafting on the contralateral side of the treated scar. Healthy skin measurements will serve as a reference (i.e. the optimum skin mechanics within that person).

\section{Scar color and pigmentation}

Scar color and pigmentation will be measured with the DSM II Colormeter ${ }^{\circledR}$ (Cortex Technology, Hadsund, Denmark), a validated and reliable tool that calculates an erythema and melanin index as a proxy for color and pigmentation (Figure 2 and 7). Measurements of the selected scar and adjacent normal skin will be performed pre-, 3- and 12-months post-tSVF-enriched grafting ${ }^{31}$.

\section{Scar quality}

Scar quality will be assessed by the POSAS version 2.029,30. The POSAS Patient and Observer scales are completed pre- 3 and 12 months post-tSVF-enriched grafting (Figure 2 and 8). All items of the POSAS 2.0 will be scored on a 10-point rating scale and added together to obtain a final score. Additionally, item specific scores and the overall opinion of the scar will be scored (Figure 8).

\section{Standardized photographs}

A standardized photograph will be taken from each scar within 24 hours before treatment under the same light and camera conditions each time. 
For participants in our study, scar biopsies are optional. If patients consent to biopsies, histological features will be assessed by taking a $2 \mathrm{~mm}$ biopsy of the selected scar on a fixed position; $1 \mathrm{~cm}$ left from point 1 within 24 hours before treatment and at 12 months post tSVF-enriched grafting $1 \mathrm{~cm}$ right from point 1 (Figure 2). Skin sample will be fixed with $2 \%$ PFA and paraffin embedded. From each patient, 0.5 $\mathrm{ml}$ of tSVF will be $2 \%$ paraformaldehyde (PFA) fixed. Fixed samples will be transported to the University and Medical Center Groningen for histological analysis. There, thin sections of paraffin embedded scar biopsies and tSVF will be deparaffinized and stained with H\&E, Picrosirius red (extracellular matrix collagen), CD68 (macrophage infiltration), perilipin A antibodies (presence of residual adipocytes), alphasmooth muscle actin antibodies (smooth muscle cells and myofibroblasts), and von Willebrand Factor and/or CD31 (endothelial cells). Stained sections will be scanned and subjected to image densitometry for quantification.

\section{Baseline patient and scar characteristics}

Patients' baseline characteristics will be extracted from patients' medical records and consist of the following: age, sex, skin type (Fitzpatrick), scar characteristics e.g. scar age, cause of the scar, prior surgery of the selected scar other than fat grafting (exclusion criterium), e.g. split skin grafting.

\section{Sample size calculation}

The primary outcome is the difference in pliability measured by the Cutometer (parameter Ue) between pre- and 12 months post-tSVF-enriched fat grafting. The expected effect size is based on the results of the study by Jaspers et al. on the Coleman fat grafting technique ${ }^{31}$. In this study, scar pliability improved by $22.5 \%$. The mean pliability measure was 0.51 . At least this effect is expected to be found in tSVFenriched fat grafting as well. An additional positive effect of $20 \%$ of tSVF-enriched fat grafting (compared to the Coleman technique) is considered a clinically relevant improvement of treatment in this study. Therefore, a sample size calculation was made with an expected effect size of 0.45 . With a required minimum of $80 \%$ power, a two tailed test and $p<0,05$ considered statistically significant the required sample size is 41 patients ( $G *$ Power 3 version 3.1.9.2). With an anticipated maximum dropout rate of $10 \%$ at 12 months, we will need to include 46 patients to compensate for drop-out.

\section{Statistical analysis}

Comparisons will be made for all primary and secondary outcome metrics of the selected scar and adjacent normal skin across all follow up moments, thus pre, 3- and 12-months post tSVF-enriched grafting, according to the established data collection protocol ${ }^{31}$. Analyses will be performed using SPSS Statistics, version 27.0 (IBM Corp., Armonk, NY) and Image J (NIH, Behesda, ML) for histological examination of skin biopsies. If data is normally distributed, results will be presented as mean with standard deviation and the primary outcome will be analyses with the paired T-test. Furthermore, the repeated measures ANOVA will be performed to detect any overall differences between related means 
across all time points. If the repeated measures ANOVA is statistically significant, we will run appropriate post hoc tests to highlight exactly where differences occur. If data is not normally distributed, appropriate non-parametric analyses are performed. Data will be analyzed using SPSS and for histological analysis, Graphpad Prism (version 8.4; GraphPad Software, Inc., La Jolla, United States) will be used. P-values $<0.05$ will be considered statistically significant.

\section{Discussion}

The aim of this prospective intra-patient before-after cohort study is to assess the efficacy of tSVFenriched fat grafting on the pliability of adherent scars caused after burn injury or fasciitis necroticans or degloving injury. The potential therapeutic capability of tSVF in adherent scars will be measured by validated objective and subjective scar assessment tools.

This proposed prospective study design is part of a larger series of consecutive prospective cohort studies with identical study design and standardized algorithm and outcome assessment scheme to allow for identical scar follow-up ${ }^{31}$. The only difference between these studies is the fat grafting technique. These series of studies aim to determine the optimal (fat grafting) treatment for patients with adherent scars. Through the same study design, core outcome set and patient populations (same inclusion criteria), the efficacy of the various techniques can be systematically compared. Moreover, it will enable historical cohort comparison over time. In this type of cohort series, as compared to classical randomized controlled trials (RCTs), no limiting inclusion criteria are used which provides a better representation of the true patient population to our opinion (real world evidence). This in contrast to RCTs in which study populations often only present a small part of the true population due to strict in- and exclusion criteria (e.g. exclusion of comorbidities). However, a limiting factor of this study is the lack of a direct comparison of two or more fat grafting techniques under the exact same circumstances as is the case in a RCT. Nonetheless, the real-world data setting that this prospective cohort study provides may result in evidence of the efficacy of tSVF-enriched fat grafting in scar treatment.

In this study, the chosen primary outcome parameter is scar pliability measured by the Cutometer. This scar pliability assessment is a reliable, non-invasive and painless parameter to assess the therapeutic effects of fat grafting as scar remodeling treatment and the cutometer is also one of the most researched validated measurement device to assess tissue pliability and elasticity ${ }^{40}$. Apart from the assessment of objective scar tissue features, inclusion of subjective patient measurements in scar assessment is essential. Subjective scar features are assessed using the validated and world-wide used POSAS questionnaires ${ }^{26,41}$. Besides the standardized protocol and core outcome set, the power of our trial design is also the proposed combination of clinical and histological analysis of tSVF treated scar samples. Together they offer great potential to measure therapeutic efficacy in a real world setting. This combination will also provide insight in the underlying mechanism of potential regenerative effects of tSVF on scar tissue, which may have direct implications for future research and clinical applications. Moreover, the long follow up period will reassure that fat, and possibly also tSVF, have initiated 
regenerative processes and scars have had allowance to reverse to healthier dermal tissue so that this can be objectified with the data outcome set ${ }^{42}$.

To ensure equal quality of treatment in the participating centers of this multicenter study and to minimize the effect of confounding factors, several standardization measures were applied. For example, specific durations have been set for procedural steps, e.g. 80 minutes for normal fat grafting procedures and 20 minutes for concentrating and injection of SVF-enriched fat grafts. Furthermore, all fat grafting procedures will be carried out according to the Coleman technique and centers will use the same fractionators to manufacture tSVF. Finally, only plastic surgeons with significant experience with fat grafting procedures will perform the surgeries to ensure equal standard of care.

Patients of the participating Dutch Burn Centers possibly provide an accurate model for burn patients and patients with adherent scars in most high-income countries and the surgical procedure is easily replicable in other like clinical environments where fat grafting can be carried out. This may improve the generalizability of the studies' conclusions. We believe that our prospective multicenter cohort study design is sufficiently powerful to provide such potential evidence that may alter the standard of care. This has precedent, for instance in the prospective cohort study of Jaspers et al, after which fat grafting for adherent scars became insured healthcare in the Netherlands.

In summary, our prospective study will contribute to further optimizing fat grafting treatments of patients with adherent scars and may potentially lead to better and more optimal treatment options for adherent scars than other fat grafting techniques.

\section{Trial Status}

This study design is a summary with backgrounds and explanations of the CCMO approved protocol (version 2.0, 27 August 2020 NL72094.000.20) and complies with the guideline of Good Clinical Practice (GCP). Inclusion opens at December 2021 and is expected to be completed in October 2024.

\section{Abbreviations}

ASC $\quad$ Adipose-derived Stromal Cells

CCMO National Dutch Ethical Committee

cSVF cellular Stromal Vascular Fraction

FAT Fractionation of Adipose Tissue

MSS Manchester Scar Scale

POSAS Patient and Observer Scar Assessment Scale 
SVF Stromal Vascular Fraction

tSVF tissue Stromal Vascular Fraction

VSS Vancouver Scar Scale

\section{Declarations}

\section{Consent for publication}

Not applicable.

\section{Availability of data and materials}

Not applicable.

\section{Competing interest}

The authors declare that they have no competing interests.

\section{Funding}

This study is partially funded by the Netherlands Organisation for Health Research and Development (ZonMw), project number: 427002011, and the Dutch Burn Foundation.

\section{Authors' contribution}

All authors contributed to the design of this study. LV and JAD drafted the manuscript. AP performed the sample size calculation and designed the statistical analysis. AP, MN, PPMZ, MCH and BL assisted to draft this manuscript. All authors reviewed the manuscript, provided comments and approved the manuscript.

\section{Acknowledgements}

We would like to thank the Netherlands Organisation for Health Research and Development (ZonMw), and the Dutch Burn Foundation for their financial contribution.

\section{References}

1. Neuber G. Fettransplantation. Chir Kongr Verhandl Deutsche Gesellschaft für Chir 1893;22:66.

2. Coleman SR. Hand rejuvenation with structural fat grafting. Plastic and reconstructive surgery. 2002;110(7):1731-1744; discussion 1745-1737.

3. Coleman SR. Structural fat grafting: more than a permanent filler. Plastic and reconstructive surgery. 2006;118(3 Suppl):108s-120s. 
4. Zuk PA, Zhu M, Mizuno $\mathrm{H}$, et al. Multilineage cells from human adipose tissue: implications for cellbased therapies. Tissue engineering. 2001;7(2):211-228.

5. Klinger $M$, Marazzi $M$, Vigo $D$, Torre $M$. Fat injection for cases of severe burn outcomes: a new perspective of scar remodeling and reduction. Aesthetic plastic surgery. 2008;32(3):465-469.

6. Aronowitz JA, Lockhart RA, Hakakian CS. Mechanical versus enzymatic isolation of stromal vascular fraction cells from adipose tissue. SpringerPlus. 2015;4:713.

7. van Dongen JA, Stevens HP, Parvizi M, van der Lei B, Harmsen MC. The fractionation of adipose tissue procedure to obtain stromal vascular fractions for regenerative purposes. Wound repair and regeneration: official publication of the Wound Healing Society [and] the European Tissue Repair Society. 2016.

8. Bourin P, Bunnell BA, Casteilla L, et al. Stromal cells from the adipose tissue-derived stromal vascular fraction and culture expanded adipose tissue-derived stromal/stem cells: a joint statement of the International Federation for Adipose Therapeutics and Science (IFATS) and the International Society for Cellular Therapy (ISCT). Cytotherapy. 2013;15(6):641-648.

9. van Dongen JA, Tuin, A. J., Spiekman, M., Jansma, J., van der Lei, B., Harmsen, M. C.,. Comparison of intraoperative procedures for isolation of clinical grade stromal vascular fraction for regenerative purposes: a systematic review Journal of tissue engineering and regenerative medicine.

10. Negenborn VL, Groen JW, Smit JM, Niessen FB, Mullender MG. The Use of Autologous Fat Grafting for Treatment of Scar Tissue and Scar-Related Conditions: A Systematic Review. Plastic and reconstructive surgery. 2016;137(1):31e-43e.

11. Spiekman M, van Dongen, J. A., Willemsen, J. C., Hoppe, D. L., van der Lei, B., Harmsen, M. C. The power of fat - emerging concepts for fibrotic scar treatment. Journal of tissue engineering and regenerative medicine.

12. Balkin DM, Samra S, Steinbacher DM. Immediate fat grafting in primary cleft lip repair. Journal of plastic, reconstructive \& aesthetic surgery: JPRAS. 2014;67(12):1644-1650.

13. Bollero D, Pozza S, Gangemi EN, et al. Contrast-enhanced ultrasonography evaluation after autologous fat grafting in scar revision. I/ Giornale di chirurgia. 2014;35(11-12):266-273.

14. Bruno A, Delli Santi G, Fasciani L, Cempanari M, Palombo M, Palombo P. Burn scar lipofilling: immunohistochemical and clinical outcomes. The Journal of craniofacial surgery. 2013;24(5):18061814.

15. Guisantes E, Fontdevila J, Rodriguez G. Autologous fat grafting for correction of unaesthetic scars. Annals of plastic surgery. 2012;69(5):550-554.

16. Maione $L$, Memeo A, Pedretti L, et al. Autologous fat graft as treatment of post short stature surgical correction scars. Injury. 2014;45 Suppl 6:S126-132.

17. Mazzola IC, Cantarella G, Mazzola RF. Management of tracheostomy scar by autologous fat transplantation: a minimally invasive new approach. The Journal of craniofacial surgery. 2013;24(4):1361-1364. 
18. Pallua N, Baroncini A, Alharbi Z, Stromps JP. Improvement of facial scar appearance and microcirculation by autologous lipofilling. Journal of plastic, reconstructive \& aesthetic surgery: JPRAS. 2014;67(8):1033-1037.

19. Phulpin B, Gangloff P, Tran N, Bravetti P, Merlin JL, Dolivet G. Rehabilitation of irradiated head and neck tissues by autologous fat transplantation. Plastic and reconstructive surgery. 2009;123(4):1187-1197.

20. Ribuffo D, Atzeni M, Guerra M, et al. Treatment of irradiated expanders: protective lipofilling allows immediate prosthetic breast reconstruction in the setting of postoperative radiotherapy. Aesthetic plastic surgery. 2013;37(6):1146-1152.

21. Sardesai MG, Moore CC. Quantitative and qualitative dermal change with microfat grafting of facial scars. Otolaryngology-head and neck surgery: official journal of American Academy of Otolaryngology-Head and Neck Surgery. 2007;137(6):868-872.

22. Wang G, Ren Y, Cao W, Yang Y, Li S. Liposculpture and fat grafting for aesthetic correction of the gluteal concave deformity associated with multiple intragluteal injection of penicillin in childhood. Aesthetic plastic surgery. 2013;37(1):39-45.

23. Zellner EG, Pfaff MJ, Steinbacher DM. Fat grafting in primary cleft lip repair. Plastic and reconstructive surgery. 2015;135(5):1449-1453.

24. Krakowski AC, Totri CR, Donelan MB, Shumaker PR. Scar Management in the Pediatric and Adolescent Populations. Pediatrics. 2016;137(2):e20142065.

25. Brown BC, Moss TP, McGrouther DA, Bayat A. Skin scar preconceptions must be challenged: importance of self-perception in skin scarring. Journal of plastic, reconstructive \& aesthetic surgery: JPRAS. 2010;63(6):1022-1029.

26. Carrière ME, Kwa KAA, de Haas LEM, et al. Systematic Review on the Content of Outcome Measurement Instruments on Scar Quality. Plast Reconstr Surg Glob Open. 2019;7(9):e2424. Published 2019 Sep 30. doi:10.1097/GOX.0000000000002424

27. Brusselaers N, Pirayesh A, Hoeksema H, Verbelen J, Blot S, Monstrey S. Burn scar assessment: A systematic review of objective scar assessment tools. Burns: journal of the International Society for Burn Injuries. 2010;36(8):1157-1164.

28. Sullivan T, Smith J, Kermode J, Mclver E, Courtemanche DJ. Rating the burn scar. J Burn Care Rehabil. 1990;11(3):256-60.

29. van de Kar AL, Corion LU, Smeulders MJ, Draaijers LJ, van der Horst CM, van Zuijlen PP. Reliable and feasible evalua- tion of linear scars by the Patient and Observer Scar Assess- ment Scale. Plast Reconstr Surg 2005;116:514-22.

30. www.posas.org

31. Jaspers ME, Brouwer KM, van Trier AJ, Groot ML, Middelkoop E, van Zuijlen PP. Effectiveness of Autologous Fat Grafting in Adherent Scars: Results Obtained by a Comprehensive Scar Evaluation Protocol. Plastic and reconstructive surgery. 2017;139(1):212-219. 
32. Verhaegen, Pauline D. H. M.; van der Wal, Martijn B. A.; Middelkoop, Esther; van Zuijlen, Paul P. M. (2011). Objective Scar Assessment Tools: A Clinimetric Appraisal. Plastic and Reconstructive Surgery, 127(4), 1561-1570.doi:10.1097/prs.0b013e31820a641a

33. Draaijers LJ, Tempelman FR, Botman YA, Kreis RW, Mid- delkoop E, van Zuijlen PP. Colour evaluation in scars: Tri- stimulus colorimeter, narrow-band simple reflectance meter or subjective evaluation? Burns 2004;30:103-107.

34. Verhaegen, Pauline D. H. M.; van der Wal, Martijn B. A.; Middelkoop, Esther; van Zuijlen, Paul P. M. (2011). Objective Scar Assessment Tools: A Clinimetric Appraisal. Plastic and Reconstructive Surgery, 127(4), 1561-1570.doi:10.1097/prs.0b013e31820a641a

35. Draaijers JL, Botman YA, Tempelman FR, Kreis RW, Middelkoop E, van Zuijlen PP. Skin elasticity meter or subjective evaluation of scars: A reliability assessment. Burns 2004;30: 109-114

36. Fong SS, Hung LK, Cheng JC. The cutometer and ultra- sonography in the assessment of postburn hypertrophic scar: A preliminary study. Burns 1997;23(Suppl 1):S12-S18.

37. Clarys P, Alewaeters K, Lambrecht R, Barel AO. Skin color measurements: Comparison between three instruments: The Chromameter(R), the DermaSpectrometer(R) and the Mexameter(R). Skin Res Technol. 2000;6:230-238.

38. https://www.trialregister.nl/trial/8461

39. Van Dongen JA, Gostelie OFE, Vonk LA, De Bruijn JJ, Van Der Lei B, Harmsen MC, Stevens HP. Fractionation of Adipose Tissue Procedure With a Disposable One-Hole Fractionator. Aesthet Surg J. 2020 Mar 23;40(4):NP194-NP201. doi: 10.1093/asj/sjz223. PMID: 31402379.

40. van der Wal M, Bloemen $M$, Verhaegen $P$, Tuinebreijer $W$, de Vet $H$, van Zuijlen $P$, et al. Objective color measurements: clinimetric performance of three. Devices on normal skin and scar tissue. J Burn Care Res 2013;34:e187-94.

41. Carrière ME, Kwa KAA, de Haas LEM, et al. Systematic Review on the Content of Outcome Measurement Instruments on Scar Quality [published correction appears in Plast Reconstr Surg Glob Open. 2019 Sep 24;8(5):e2933]. Plast Reconstr Surg Glob Open. 2019;7(9):e2424. Published 2019 Sep 30. doi:10.1097/GOX.0000000000002424

42. Spiekman, M, Francia, DL, Mossel, DM, Brouwer, LA, Diercks, GFH, Vermeulen, KM, Folkertsma, M, Ghods, M, Kzhyshkowska, J, Klüter, H, Krenning, G, van der Lei, B, \& Harmsen, MC (2021). Autologous lipofilling improves clinical outcome in patients with symptomatic dermal scars through induction of a pro-regenerative immune response. Aesthetic Surgery Journal. Htpp://doi.org/10.1093/asj/sjab280

\section{Figures}


Patient meets inclusion criteria
Start of the study

Patient intake at one of the hospitals
Within 24 hours before treatment: Standardized photo T1, Cutometer T1 + DSM Colormeter T1 + POSAS T1
Standardized photo T2, Cutometer T2 + DSM Colormeter T2 + POSAST2
Standardized photo T3, Cutometer T3 + DSM Colormeter T3 + POSAS T3
Patient information handed out

Patient agrees to participate in the study

Study number assigned to patient

Scar selected for experimental treatment

Optional: One $2 \mathrm{~mm}$ biopsy (into the dermal fat) taken from standardized place in the selected scar after local anesthesia

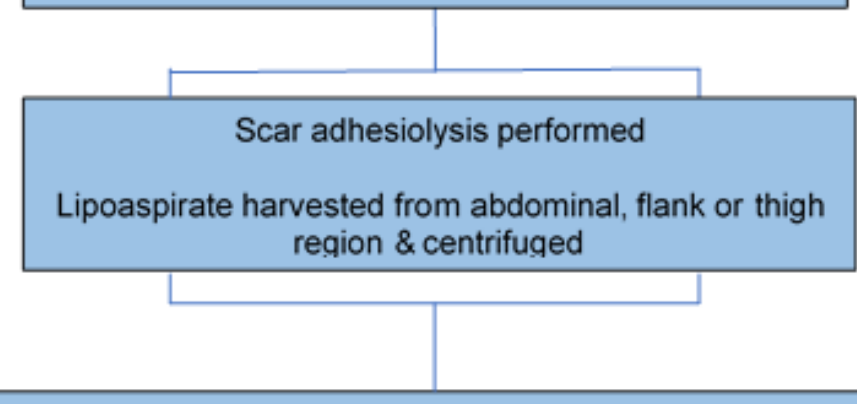

Fat mechanically dissociated \& centrifuged, resulting in tSVF

tSVF mixed with fat graft

tSVF enriched fat graft injected under selected scar

Per patient, $0,5 \mathrm{ml}$ of SVF is sent to UMCG lab for analysis
3 months (+/- 2 weeks) postoperative: Study measurements (details on the left)

$$
\text { Check-up; standard of care }
$$

12 months (+/- 1 month) postoperative: Study measurements (details on the left) Optional: One $2 \mathrm{~mm}$ biopsy (within area of the selected scar) taken (into the dermal fat) under local anesthesia.

\section{Figure 1}

Prospective cohort study design 


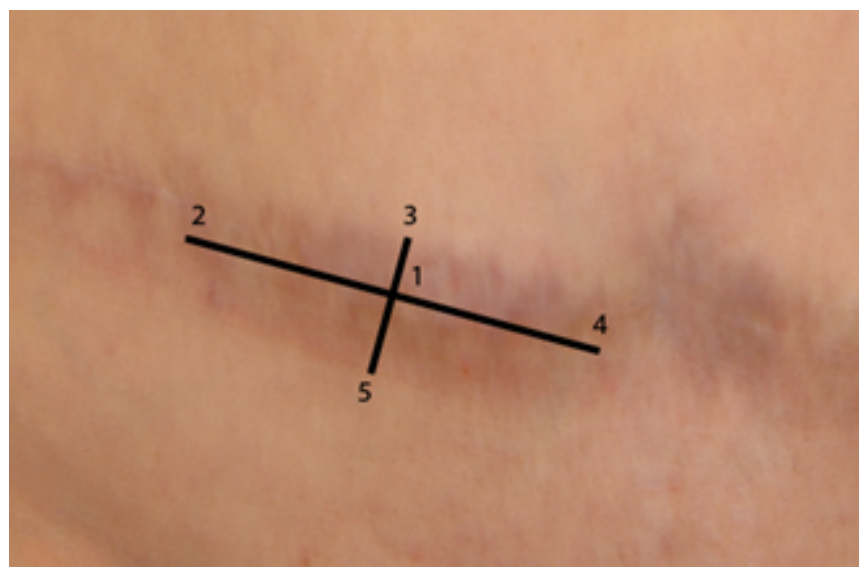

Figure 2

Selected scar divided in 5 points according to a standardized algorithm
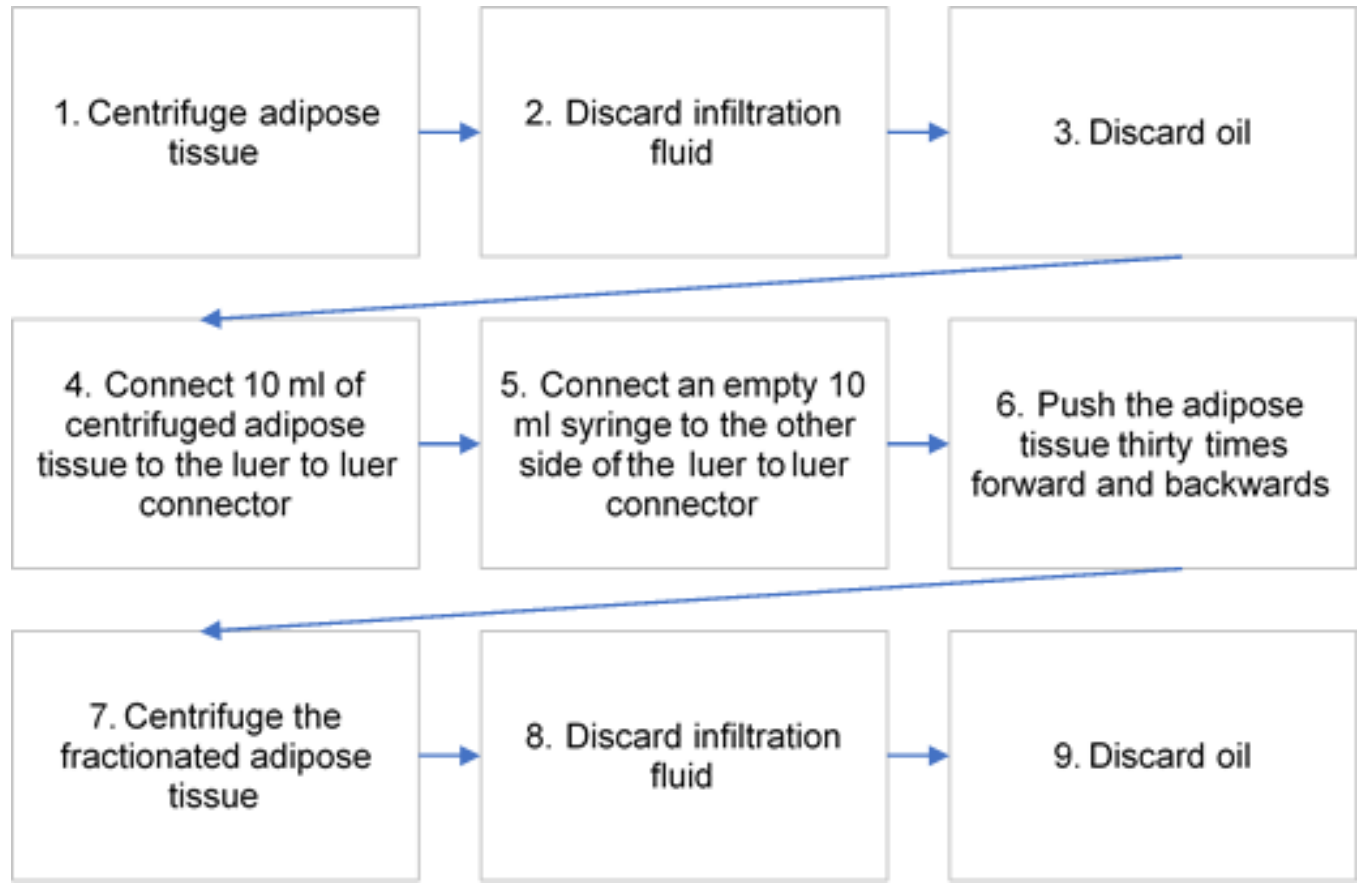

\section{Figure 3}

Flow diagram of Fractionation of Adipose Tissue procedure (FAT procedure) 


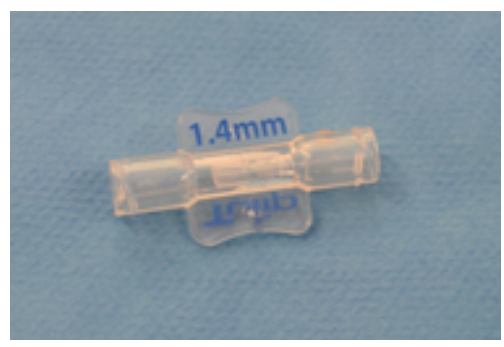

\section{Figure 4}

Frontal-and side view of disposable fractionator with one hole of $1.4 \mathrm{~mm}$ inside, used to fractionate adipose tissue

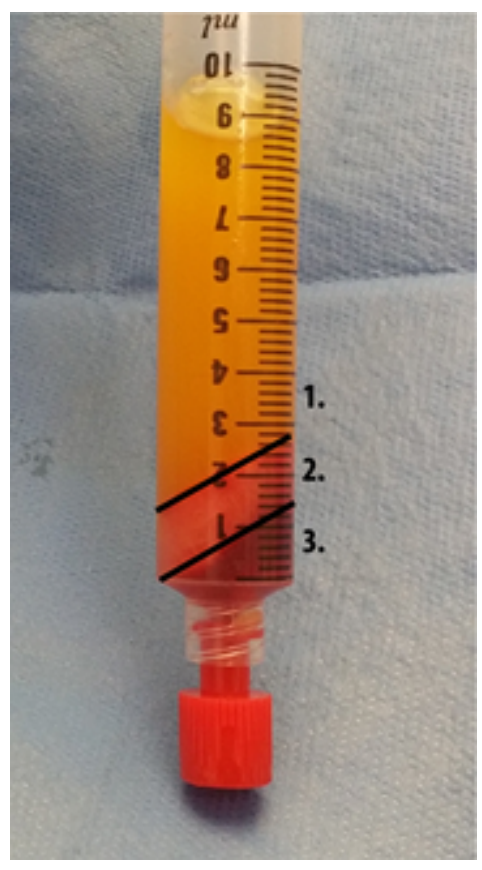

\section{Figure 5}

Lipoaspirate after performing the Fractionation of Adipose Tissue procedure

1) Oil = disrupted adipocytes 2) tissue Stromal Vascular Fraction 3) Infiltration fluid including pellet consisting of dead cell remainders 


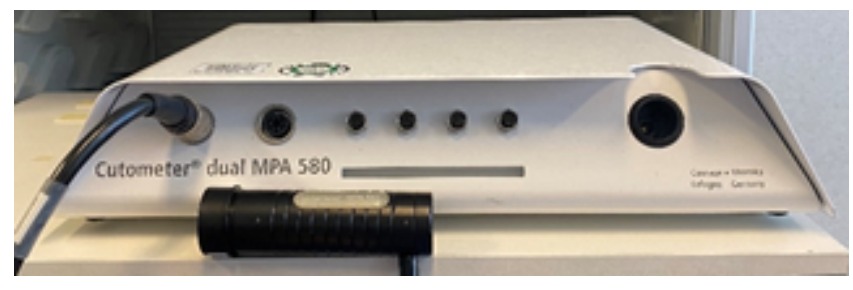

\section{Figure 6}

The Cutometer measures scar mechanics retraction, elasticity, viscoelasticity and maximum extension by inducing negative pressure on scar tissue (Cutometer Skin Elasticity Meter Dual MPA $580 \AA$ Courage and Khazaka GmbH, Cologne, Germany)

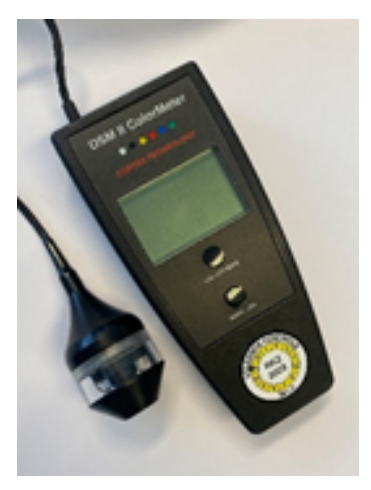

Figure 7

The DSM II Colormeter measures scar color and pigmentation by calculating an erythema and melanin index (Cortex Technology, Hadsund, Denmark)

\section{Figure 8}

The POSAS Scale 2.0. The patient score combines scar pain, itch, color, stiffness, thickness and irregularity. The observer scale comprises of the items vascularity, pigmentation, thickness, relief, pliability and surface area. All items of the patient and observer score combined will form a total POSAS score (the highest score represents the worst scar imaginable). It also consists of an overall opinion of the scar. 